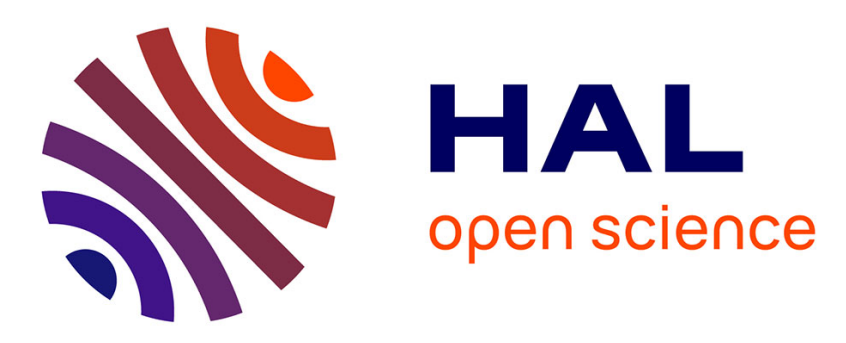

\title{
Using facial expressiveness of a virtual agent to induce empathy in users
}

\author{
Anne-Sophie Milcent, Abdelmajid Kadri, Simon Richir
}

\section{To cite this version:}

Anne-Sophie Milcent, Abdelmajid Kadri, Simon Richir. Using facial expressiveness of a virtual agent to induce empathy in users. International Journal of Human-Computer Interaction, 2021, 10.1080/10447318.2021.1938387 . hal-03275590

\section{HAL Id: hal-03275590 \\ https://hal.science/hal-03275590}

Submitted on 1 Jul 2021

HAL is a multi-disciplinary open access archive for the deposit and dissemination of scientific research documents, whether they are published or not. The documents may come from teaching and research institutions in France or abroad, or from public or private research centers.
L'archive ouverte pluridisciplinaire HAL, est destinée au dépôt et à la diffusion de documents scientifiques de niveau recherche, publiés ou non, émanant des établissements d'enseignement et de recherche français ou étrangers, des laboratoires publics ou privés. 


\title{
Using facial expressiveness of a virtual agent to induce empathy in users
}

\author{
Anne-Sophie Milcent ${ }^{\mathrm{a}}$, Abdelmajid Kadri ${ }^{\mathrm{a}}$ and Simon Richir ${ }^{\mathrm{a}}$ \\ a Arts et Metiers Institute of Technology, LAMPA, HESAM Université, Rue Marie Curie, \\ 53810 Changé, France;
}

\begin{abstract}
Healthcare simulators are learning environments that offer many training opportunities. The integration of expressive virtual patients in these simulators encourages the exchanges and provokes emotional reactions in the learner, which promotes memorization and learning. Based on these elements, we assume that the facial expressiveness of a virtual agent is a factor to be considered in order to improve the user experience. We investigate the impact of the facial expressiveness of virtual humans on the empathy induction in the user of health simulators. We also investigate the impact of facial expressiveness on the user's sense of similarity and the affective bond with the agent. Our results show a high empathy score when users train with simulators. Depending on the context, we observe a significant difference in perspective-taking in favor of the users who interact with an expressive virtual agent to a virtual agent without facial expressiveness.
\end{abstract}

\section{KEYWORDS}

Virtual agent ; empathy ; facial expressiveness ; user experience ; health.

\section{Introduction}

Aware of living a virtual experience, users are still affected by the narrative elements present in the virtual experience, which is why they can develop empathy for the virtual agents. They consider that potentially these agents, help them, share their goals and expectations (Paiva, Leite, Boukricha, \& Wachsmuth, 2017). Perron (2005) defines fictional emotions as emerging from "the comparison of magnified fictional events with the emotional potential of comparable events in everyday life". It is this sharing and appropriation of objectives that make emotions possible. Fictional empathy comes from an identification with the situation and the character as well as concern and attachment to him.

The relationship between virtual agents and the user has been the subject of several types of research, to improve human-computer interactions and increase the user's emotional involvement, leading to better memorization (Tyng, Amin, Saad, \& Malik, 2017; Veletsianos, 2009). Connecting and building relationships with virtual agents is one of the key challenges to improving the user experience. In our research, we study the induction of empathic reactions in the user, towards virtual agents, within a 
simulator for healthcare professional training. In this study, we also analyze the impact on the affective bond and the feeling of similarity felt by the user.

The implementation of an empathic situation was achieved through to the empathy factors described by Paiva et al. (2017). We took into consideration the situation and context (medical environment and scenario), the mechanisms of empathy and the characteristics of the user. Finally, we worked on the characteristics related to the virtual agent: realistic appearance, behaviors, dialogues, expressiveness. The implementation of an empathic situation allowed the design of an appropriate experiment to study the impact of the virtual agent's facial expressiveness on the induction of user empathy. During the simulation, the user is confronted with a virtual agent with or without emotional facial expressions, depending on the conditions of the experiment.

Section 2 presents a synthetic state of the art concerning the theories of empathy, the implementation of an empathic situation and the characteristics of the virtual agent for the induction of empathy. Section 3 describes the experimentation. In Section 4, results are analyzed, then implications are discussed in Section 5. Section 6 concludes the study. Section 7 is dedicated to acknowledgments.

\section{Related Work}

\subsection{Empathy}

Empathy is commonly described as the ability to put oneself in the other's place. In reality, empathy is more complex. It is divided into two components: affective and cognitive (Davis, 1983). The first component corresponds to the cognitive ability to take the subjective perspective of others (cognitive empathy). The second component is an emotional response towards others that sometimes involves sharing one's emotional state (affective empathy).

Affective empathy is a neurological approach. It refers to an individual's empathic reactions to the emotional state of others. The perception of the emotional state of others activates in the individual a representation of this state, as a resonance system to understand what others are doing or feeling (Narme et al., 2010). Affective empathy can be articulated around emotional resonance: imitation (Hess \& Fischer, 2014; Iacoboni, 2009) and emotional contagion. The individuals imitate and synchronize their facial expressions and posture according to those of others. The tendency to imitate is to react emotionally to the facial expressions of other (MacDonald, 2003). The emotional contagion means the automatic mirroring of the emotional state of others (Rizzolatti \& Sinigaglia, 2008; Wicker et al., 2003).

Cognitive empathy is a psychological approach to empathy. It corresponds to the understanding of the emotional state of other (Guillain \& Pry, 2012). Cognitive empathy means being able to adopt the perspective of others (Berthoz \& Jorland, 2004; Decety, 2002). Unlike affective empathy, this projection is not automatic but controlled. Perspective-taking is defined as a tendency to adopt the psychological point of view of others. It is also related to the theory of mind, i.e. the ability to understand and predict the behavior and emotions of others based on their knowledge, intentions, desires, beliefs, etc. This corresponds to the ability to recognize our own mental state, but also the mental state of another individual. Cognitive empathy, including the theory of mind, is important for good human interactions because the ability to understand others' mental states is an essential aspect of social intelligence (Frith \& Frith, 1999, 2005). Furthermore, Reniers, Corcoran, Drake, Shryane, and Völlm (2011) refers to 
the notion of simulation when defining cognitive empathy. This mental simulation corresponds to a projection of oneself in the place of others through the imagination. Finally, empathy has a central role in social interactions (Narme et al., 2010).

\subsection{Empathic situation}

An empathic situation integrates an "observer" (the user), who responds emotionally to the target's emotional state. This target, i.e. the virtual agent in this study, expresses an emotional state, or is in a situation perceived as emotional by the user (Paiva et al., 2017). There are different notions involved in an empathic situation between a user and a virtual agent. Paiva et al. (2017) investigated these notions that promote the creation of an empathic situation:

(1) context and situation;

(2) empathy mechanisms;

(3) user characteristics;

(4) empathy modulation;

(5) virtual agent characteristics.

First of all, the situation implies that an event has occurred and that the observer has witnessed it (directly or indirectly). This results in an emotion, which affects the user. This emotion can be felt by the target or simply imagined by the user as being felt by the target (Paiva et al., 2017). It is also necessary to take into account the duration of the situation, the desirability of the event or the presence of other individuals during this event.

Concerning empathy mechanisms, interaction with a virtual agent can affect users' emotions. The situation can encourage users to project themselves in the agent's place based on a cognitive mechanism related to perspective-taking. The agent's expression can also trigger affective empathy through an automatic imitation and feedback phenomenon (Boukricha, 2013; Paiva, Dias, Sobral, Woods, \& Hall, 2004; Paiva et al., 2017). The empathic response of the user is influenced by the emotion expressed by the agent. Rodrigues, Mascarenhas, Dias, and Paiva (2015) characterizes the emotion expressed according to its type (joy, anger, etc.), valence (positive or negative emotion), intensity (degrees of emotion), and cause (related to a triggering event). Each of these emotional characteristics can influence the intensity of the user's empathic responses.

In addition, the user's characteristics can also influence the empathic situation. These factors include age, gender, past experiences, culture, ability to establish or maintain a social relationship and personal differences (such as illness) (Davis, 1983; De Vignemont \& Singer, 2006).

Concerning the empathy modulation, as in human interactions, observers may have more or less intense empathic reactions depending on the situation and the target (De Vignemont \& Singer, 2006). They are influenced by different modulating factors such as the feeling of similarity, the emotional relationship between the agent and the user, the personality or the mood of the user (Davis, 1983; Hoffman, 2001). Within their work on empathic agents, Rodrigues et al. (2015); Rodrigues, Mascarenhas, Dias, and Paiva (2009) describe some of these modulation factors. The emotional link corresponds to the relationship with the virtual agent, the affection they have for each other. This link reinforces or decreases the intensity of the empathic response that will be provided. 
Mood represents the emotional state that will influence the empathic response. For example, a good mood will increase the chance of getting a positive empathic response. The personality is also one of the characteristics that modulate the empathic response. Finally, the greater the similarity (emotional, behavioral or visual resemblance between the user and the agents), the stronger the empathic response will be.

The last notion involved in an empathic situation between a user and a virtual agent : characteristics of the virtual agent for the induction of empathy will be described in the following section.

\subsection{Induction of empathy: importance of the virtual agent's characteristics}

Among the notions that promote the development of an empathic situation, we have previously described the mechanisms and modulation of empathy, the characteristics related to the users and the context. But to induce empathic reactions in the simulator, particular attention is also required regarding the characteristics of the virtual agent. These characteristics include the agent's appearance (realism), his behavior, the experience and the intellectual capacities attributed to him through the scenario, as well as his expressiveness (McDonnell, Breidt, \& Bülthoff, 2012; Zell, Zibrek, \& McDonnell, 2019; Zibrek, Martin, \& McDonnell, 2019).

The users and their experience are affected by the realism of virtual agents and the emotions they convey (Beale \& Creed, 2009; Guadagno, Blascovich, Bailenson, \& McCall, 2007; Guo, Mingming, Qingwing, \& Duffy, 2019). Several researchers have made recommendations on the visual realism of agents and their impacts (Zell et al., 2015, 2019). Studies have shown that photo-realistic agents are appreciated and have an impact on the user (Chaturvedi, Newsome, \& Babu, 2015; McDonnell et al., 2012). There is nevertheless an undeniable risk in the search for realism: the uncanny valley that could provoke a non-acceptance of the agent (Mori, MacDorman, \& Kageki, 2012; Mori, MacDorman, \& Minato, 1970), which is why it is necessary to pay particular attention when designing virtual agents regarding their photorealistic rendering and their animation. In addition, the appearance of a virtual human and its characteristics can emotionally affect the users of the simulator (Ter Stal, Tabak, Op Den Akker, Tessa, \& Hermie, 2020), as well as their perception of the agent's personality and social characteristics. This encourages the emergence of social behaviors such as shame or shyness in the users (Volonte et al., 2016). Realistic virtual agents facilitate the establishment of a trusting relationship and influence the emotional reactions of users (Chaturvedi et al., 2015; Lemoine \& Cherif, 2012). The conception of a credible and realistic character also depends on behavior realism. The transcription of the agent's emotional and mental state is an important factor of credibility. The agent's behavior can also induce a sense of social presence in the user (Von Der Pütten, Krämer, \& Gratch, 2009), or a strong engagement (Glas \& Pelachaud, 2015; Hall, Woods, Aylett, Newall, \& Paiva, 2005). This enriches the interactions and allows a stronger emotional involvement of the user, which is necessary when using simulators dedicated to learning.

The agent's face is a major element of interaction in exchanges involving emotional reactions (Fabri \& Moore, 2005). Indeed, the presence of facial expressions positively affects the perception of the virtual agent, the motivation, the learning and the attitude of the user (Baylor \& Kim, 2009).

A study by Hasler, Spanlang, and Slater (2017) showed that non-verbal behavior 
and facial mimicry influence users. The agent's verbal and non-verbal behavior must be consistent and appropriate. Moreover, the use of expressive agents allows users to have a good understanding of their feelings and emotional state (Fabri \& Moore, 2005).

Designing an expressive virtual agent requires an understanding of the representation of human emotions. Ekman (1993) indicates that there is an innate facial expression for each basic emotion. Each emotion and expression is a combination of facial muscles and intensity levels (Ekman, 2007). Researchers have made recommendations on the transcription of emotions onto virtual humans and in particular on the characteristics of facial emotional expressions (Ochs, Niewiadomski, \& Pelachaud, 2015; Pelachaud, 2009; Vinayagamoorthy et al., 2006). The aim of this research is to improve user interaction. Indeed, Bates (1994) highlights the importance for virtual humans to be able to express emotions in order to appear credible and give the illusion of life. Facial expressions, gestures, voice, dialogue, attitude or gaze can participate in immersing the user in a strong emotional context and facilitate the appearance of emotional and empathic reactions. The transcription of emotions on a virtual agent also concerns expressive wrinkles (Courgeon, Buisine, \& Martin, 2009; Larboulette \& Cani, 2004), the gaze and variation of pupils (Garau, 2003; Li, Mao, \& Liu, 2009), the duration and intensity of emotions as well as variations in skin color (De Melo \& Gratch, 2009).

Finally, researchers Philip, Martin, and Clavel (2018); Ravaja, Bente, Kätsyri, Salminen, and Takala (2016); Weyers, Mühlberger, Hefele, and Pauli (2006) have also worked on the reactions of users when confronted with the emotional facial expressions of agents. These researches point out that there is an impact of the agent's expressivity on the user's emotional responses.

Our literature review illustrates the plurality of factors that can influence the induction of empathic reactions in the user. We purpose to pursue investigations in this direction to identify the impact of the virtual agent's facial expressivity on the user experience, especially on the induction of empathy in the user of simulators:

- Hypothesis 1: The facial expressiveness of virtual agents impacts positively the user's empathy.

- Hypothesis 1.1: Expressive virtual agents have a positive impact on the cognitive empathy of the participant.

- Hypothesis 1.2: Expressive virtual agents have a positive impact on the affective empathy of the participant.

- Hypothesis 2: Expressive virtual agents increase the user's sense of similarity and affective bond.

\section{Materials and methods}

\subsection{Application : context and virtual environment}

For this experimentation, we use a nursing simulator created by the company SimforHealth, specialized in the design of simulators for the training of healthcare professionals. The objective of this simulator is the accompaniment of a person in the practice of her daily care. In this simulator, students must take charge of a virtual patient, accompany her to the toilet and react appropriately to the pain she feels. The trainers of the Institutes of Nursing of Nouvelle-Aquitaine (France), who created the 
scenario for this simulator, provided an accurate description of the environment and the patient, hence the graphic choices made. The dialogues, interactions, and agent behavior are based on discussions with health professionals to ensure the credibility of the virtual patient. The behaviors were detailed during the scenario writing process and the animations were validated during the simulator design process. As part of this study and also in partnership with the trainers, the simulator has been adapted to induce emotions and create empathy in the users. Indeed, the induction of empathy is made possible by the context and behavior of the virtual agent, specially her dialogues, her emotional facial expressions and body language. Depending on the situation and the exchanges within the simulator, the patient expresses her emotions and her feelings (her pain, her suffering, her gratefulness, etc.). The respect of empathy factors in virtual environments has been taken into account, whether in relation to the characteristics of the patient, the situation or the interactions implemented.

The environment is important in the context of empathy simulation, that's why this entire simulator takes place in the virtual patient's room in a residential facility for dependent elderly people.

\subsection{Realistic and expressive virtual agent}

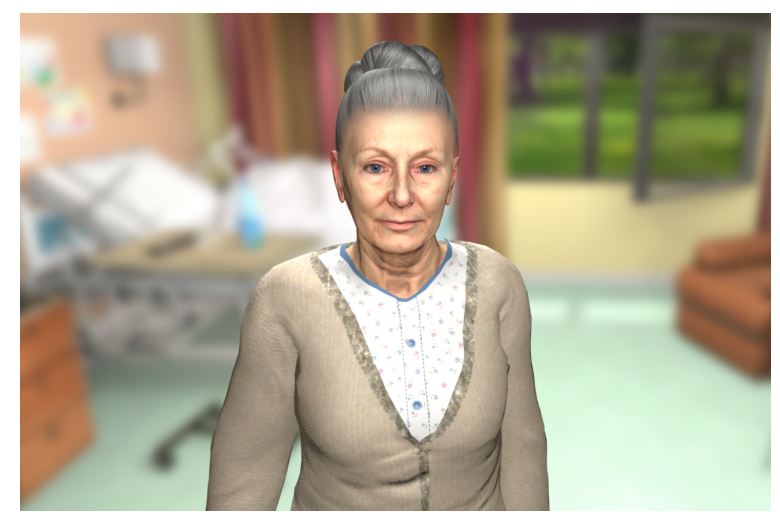

Figure 1. Realistic and expressive virtual agent

We have designed an expressive virtual human (Figure 1) using advanced modeling techniques such as photogrammetry and emotion transcription techniques: facial animations based on FACS (Ekman \& Friesen, 1978), expressive wrinkles, variation of pupils size depending on the emotional state, skin rendering (skin texture, subsurface scattering), etc. The recognition of basic emotions in this model was evaluated in a previous study (Milcent, Geslin, Kadri, \& Richir, 2019). This character has a body morphology, age, gender and clothing appropriate to the patient's description of this simulation. This agent also benefits from lip synchronization and body expressions adapted to the dialogues (nodding, breathing, hand movements).

\subsection{Materials : description of the platform}

The application is executed on a computer equipped with an Intel Core i7-6700HQ processor and a Nvidia GeForce GTX 1060 or GTX 1070 graphics card, but the difference has no impact on the rendering and application. These computers also have a 
webcam to capture the participant's facial expressions using the Affdex plugin from Affectiva ${ }^{1}$ developed for Unity. This solution is appropriate for an integration within our experimentation. It uses 34 tracking points to capture about 20 facial expressions and can detect basic emotions. According to Affectiva, the perception of these emotions by the device reaches an important accuracy threshold, about $90 \%$ (see McDuff et al. (2016) for a detailed review of the Affdex system).

The application is executed with a definition of $1920^{*} 1080$ resolution, this high definition allows good visualization of the virtual agent and her reactions.

\subsection{Participants}

52 participants were recruited for our experiment, aged 18 to 49 ( $\mathrm{M}=25.29$; $\mathrm{SD}=7.663)$. This panel was composed of 42 women $(80.8 \%$ women) and 10 men. These participants were second-year (30 students) and third-year (22 students) students from the Nursing Training Institutes of the Nouvelle-Aquitaine region (France). The diversity of our study was limited by the distribution of students in this field, women are mainly represented in this profession.

\subsection{Procedure}

In order to investigate the impact of the expressiveness of the virtual agent on empathy in the user, the two conditions dependent on the expressiveness of the agent are:

- realistic and Expressive Virtual Agent (EVA), possessing emotional facial expressions,

- realistic Virtual Agent (VA), not possessing facial expression.

The simulator is divided into two phases, called situations. The participants test the two situations successively. However, situations are different and are considered independently and separately from a pedagogical and statistical point of view. Depending on the situation, each group of participants tests one of the two conditions of the expressiveness of the virtual agent. The analysis is performed according to a between-subject design, opposing group A and group B for the same situation.

Table 1. Organization of the study

\begin{tabular}{lcc}
\hline & EVA & VA \\
\hline Situation 1 & Group A & Group B \\
\hline Situation 2 & Group B & Group A \\
\hline
\end{tabular}

${ }^{1}$ Ww. affectiva.com/ 


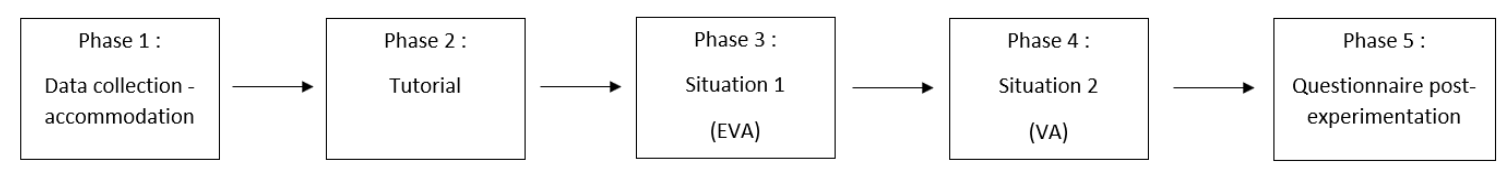

Figure 2. Description of different phases of the experiment for group A. (Group B performs the same phases, however in situation 1, group B is confronted with VA, then in situation 2 group B is confronted with EVA.)

First, each participant completes a consent form and then the experimenter invites the participant to sit down in front of the computer and provides the necessary instructions for the experiment.

\section{Phase 1: Data collection and accommodation}

During this phase, the participants fill out a pre-pass questionnaire to collect demographic information, which may provide us with information on the participants' profiles and their level of comfort with the digital tools. Next, the participants complete a questionnaire regarding their mood: the Positive Affectivity and Negative Affectivity Scale (PANAS) (Thompson, 2007; Watson, Clark, \& Tellegen, 1988). The objective is to determine if the participants are predisposed to be empathetic because the mood is one of the factors modulating empathy (Davis, 1983; De Vignemont \& Singer, 2006; Paiva et al., 2017). Finally, before beginning the experiment, the participants answer a few questions related to their empathy on a daily basis. These questions are from the Questionnaire of Cognitive and Affective Empathy (Reniers et al., 2011).

\section{Phase 2: Tutorial}

The training provided by the simulator begins during this phase. To familiarize themselves with the interface and its components, the participants visualize a short tutorial integrated in the application.

\section{Phase 3: Situation 1}

This first situation has the educational objective of measuring the patient's ability to carry out the activities of daily life such as washing and accompanying her in her care. In this situation, the learners should take charge of the patient, including talking with her and asking her about her habits and preferences. This situation involves a dialogue with two predefined exchanges and six questions for which the student must choose the most appropriate answer as a caregiver and corresponding to the needs and preferences of the virtual patient. This exercise requires the learner to think about the patient's care and psychological perspective in order to provide an appropriate response or an appropriate question. An example of questions:

You are talking to Madame Garonne. Choose the proposal which seems to you the most adequate - Question:

"-At what time do you usually wash up?"

"-I have planned to come at 9am is that ok with you?"

" - What time would you like me to come and help you wash up ?"

During this phase, the facial expressions of participants are captured through the webcam, to analyze their emotional reactions, a potential sign of affective empathy (imitation, emotional contagion). 


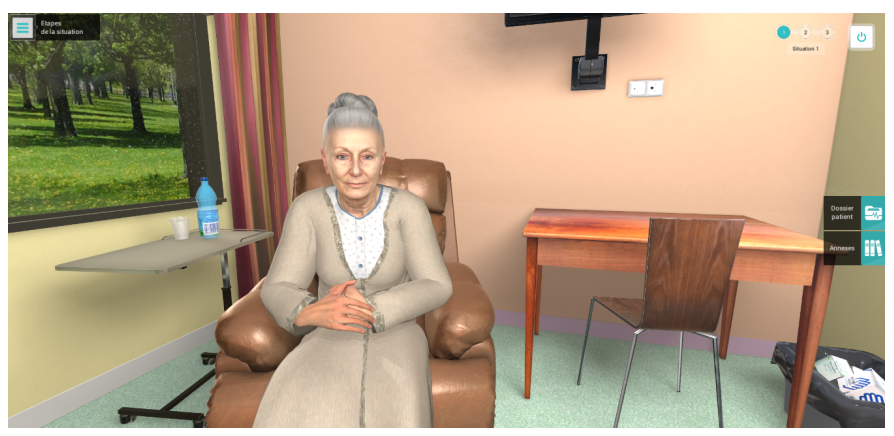

Figure 3. Exchange with the virtual patient during situation 1

At the end of situation 1, the participants answer the questions, contextualized with the study, of the Questionnaire of Cognitive and Affective Empathy (QCAE) by Reniers et al. (2011). Following the empathy questionnaire, the learners answer a question on the affective bond potentially developed towards the patient and two questions on the perceived feeling of similarity. These notions are part of the factors that modulate empathy.

\section{Phase 4: Situation 2}

Situation 2 begins with a room of errors ${ }^{2}$, then the learners exchange with the patient about her pain. The learners must take charge of the organization of the care. This situation includes a predefined dialogue of eight exchanges with the patient, as well as two questions for which they choose the most appropriate response. An example of predefined dialogues :

- User: "Have you reported it to anyone?"

- Patient: "No I thought it was because I had been sitting in my chair too long but it still hurts."

- User: "On a scale of 1 to 10, how would you rate your pain?"

- Patient: "I don't know, maybe 3."

- User: "Where does it hurt?"

- Patient: "Here in my lower back."

During this phase, facial expressions of the users are also captured. Users again complete the questionnaires related to empathy, similarity and affective bond.

In both situations, the predefined dialogues are exchanges with the patient that are activated without user intervention, while the questions require that users choose the most appropriate exchange between three or even four proposals. The first situation concerns only two predefined exchanges, but it contains several questions that require the learners to reflect on the patient's needs as well as her wishes. The learners must imagine and project themselves in order to understand the mental state of the patient. The second situation has just two questions but contains several predefined exchanges. In these predefined exchanges, users do not intervene, however, the virtual patient's state is revealed to them during the dialogue. Therefore, the two situations have different contexts and require different user involvement.

${ }^{2}$ The "room of errors" is a health simulation tool, allowing participants to practice by visualizing and identifying errors deliberately inserted in a care scene. 


\section{Phase 5: Questionnaire post-experimentation}

At the end of the simulation, participants complete a post-experimental questionnaire aimed at collecting their opinions, particularly with regard to their feelings towards virtual agents and their expressiveness. This questionnaire also includes openended questions that allow participants to freely write comments in order to share their feelings.

\subsection{Measure}

Although it is an integral part of human interactions, empathy remains a complex and difficult concept to measure. The affective component of empathy is best measured with an objective quantitative method such as psycho-physiological or behavioral measures, whereas cognitive empathy lends itself more to subjective measures. Consequently, and in order to obtain representative results, we have opted for the conjoint use of objective and subjective measures in order to obtain a maximum of information on the user's experience and to match the two components of empathy. We use two expressiveness conditions to allow a comparison between situations confronting the user with a virtual agent with emotional facial expressions (EVA) or an agent without facial expressions (VA). Participants complete identical questionnaires that will be used to study the possible impacts of facial expressiveness on the dimensions taken into account. All this data are recorded in a CSV file. The following data are collected:

- Information about each participant: gender, age, year corresponding to the course of study school.

- Participants' practices regarding digital tools: There are four items concerning the ease of participants with digital tools. Their answers are evaluated using a 5-point Likert scale. Example of items:

- I feel comfortable with digital tools (computer, phone, tablet, etc.).

- I use or have used digital simulators.

- Positive And Negative Affect Scale: The PANAS scale was defined by Watson et al. (1988). Thompson (2007) proposed a short version composed of 10 items, which we used in our study. Affectivity is related to mood. This provides measures of the positive affect (PA) and negative affect (NA) of users. These are two independent factors. A high negative affect score potentially reveals psychological distress involving a variety of unpleasant emotions, while a low score reflects calm and serenity (Watson et al., 1988). The responses to this questionnaire are evaluated with a 5-point Likert scale. This questionnaire provides an indicator of the participant's mood, which is a factor of modulation of empathy (De Vignemont \& Singer, 2006; Paiva et al., 2017).

- Cognitive and affective empathy: this questionnaire is based on other known questionnaires such as the Interpersonal Reactivity Index from (Davis, 1983) or the Empathy Quotient from (Baron-Cohen \& Wheelwright, 2004). In order to certify the reliability of the results obtained in the QCAE, the psychometric properties and validity of this questionnaire were evaluated by Reniers et al. (2011). This questionnaire has also been recently studied and validated in Portuguese study (Queirós et al., 2018), French study (Myszkowski et al., 2017) and Asian study (Liang et al., 2019).

The QCAE questionnaire is divided into five dimensions dependent on cognitive and affective empathy. The higher the score is, the more empathetic the participant feels. The questionnaire items focus on the user's daily empathy or 
contextualized in relation to the experience. These 31 items are evaluated using a 4-point Likert scale, as recommended by Reniers et al. (2011). Cognitive Empathy contains 19 items and addresses the notions of:

- perspective-taking (PT): spontaneous tendency to put oneself in the place of others, to adopt one's psychological point of view (10 items so the cumulation varies from 10 to 40 points.);

- online simulation (OS): tendency to project oneself in the place of others by imagining how one feels and one's future intentions (9 items so the cumulation varies from 9 to 36 points.);

Affective empathy concerns 12 items and addresses the notions of:

- emotional contagion (EC): automatic mirroring of the feelings of others (4 items so the cumulation varies from 4 to 16 points.);

- proximal reactivity (PRR): emotional response to the observation of the emotional state of others in a close social context (4 items so the cumulation varies from 4 to 16 points.);

- peripheral reactivity (PER): emotional response to the observation of the emotional state of others in a distant social context (4 items so the cumulation varies from 4 to 16 points.).

Examples of QCAE questionnaire items, adapted to our experimentation:

- I am good at predicting how the patient feels.

- When I am upset at the patient, I usually try to "put myself in her shoes" for a while.

- I sometimes find it difficult to see things from the patient's point of view.

- I am happy when the patient is happy, and sad when the patient is sad.

- I often get deeply involved with the feelings of a character in a film, play, or novel.

The responses from this questionnaire can be interpreted on the basis of the two main factors of empathy, affective and cognitive, as well as on the basis of the five factors described above. However, Myszkowski et al. (2017) and Queirós et al. (2018) point out that although the two-factor structure of this questionnaire is considered acceptable, the five correlated factors structure has an advantage. In fact, they recommend the use of this questionnaire taking into account all five dimensions.

- Affective bond and sense of similarity: In the literature, these notions are generally addressed by subjective evaluations such as in the study by Hall et al. (2005). We formulated two items regarding the feeling of similarity and one item concerning a potential affectivity towards the patient, related to the factors that modulate empathy. These items provide us more information about the impact of the agents' expressiveness on the users' empathic reactions. This can have an impact on the participant's empathy (Hall et al., 2005; Paiva et al., 2004). They are evaluated using a 5-point Likert scale:

- I was comfortable with this virtual patient. Her reactions seemed appropriate and natural to me.

- This virtual patient looks or might look like one of my patients.

- I enjoyed the interaction with this virtual patient.

In a second CSV file, we have collected data about facial capture. For each exchange with the patient, the file contains the question asked to the virtual patient, the type of emotional facial expressions expressed by the patient, as well as the means 
concerning the activated facial areas and emotions expressed by the user during this exchange. The objective is to determine if the participant had a facial reaction when confronted with the virtual patient's emotional state. The emotions analyzed are the basic emotions (Ekman, 2007; Ekman \& Friesen, 1978), (refer to (McDuff et al., 2016) for details of the Affdex solution).

Finally, the post-experiment questionnaire is completed by the participants to collect their subjective feelings. One of the objectives of collecting this data is to provide additional information on the participants' choices. This is intended to contribute to our discussion and to provide a more detailed analysis of the user experience.

\section{Results}

\subsection{Data analysis strategy}

First of all, we describe data about participants, such as age and gender. Then, we conduct a correlation analysis between these demographics data and the general empathy of participants during the entire experiment.

Then, we perform a detailed analysis of the empathy of the participants confronted with the expressive virtual patient (EVA) and the participants confronted with the virtual patient (VA). The differences between participant groups A and B depend exclusively on the expressiveness of the virtual patient. We begin by comparing the data of group A and group B for situation 1 by first examining the responses to the Questionnaire of Cognitive and Affective Empathy (Cognitive Empathy and Affective Empathy), then analysing the data from the facial capture (Affective Empathy) and finally analysing the data on the feeling of similarity and emotional bond. In parallel, we repeat these analyses between the different groups of participants for situation 2 which is composed of different interactions and a different pedagogical objective. Finally, we conclude with an analysis of the data collected during the post-experimental questionnaire.

\subsection{Users characteristics and general empathy}

A statistical analysis of the digital habits of the participants had been conducted. On a 5 -point Likert scale ( $5=$ strongly agree, $1=$ strongly disagree), participants are on average comfortable with digital tools such as smartphones, tablets or computers $(M$ $=4.23 ; S D=0.807)$. However, there is a low familiarity with simulators $(M=1.73$; $S D=0.744)$, assessed using a 5 -point scale $(5=$ very frequently, $1=$ never $)$. It should be noted that $86.5 \%$ of participants have reported rarely or never using simulators.

Before starting the simulator, we wanted to evaluate the participant's mood to determine if the participant is predisposed to be empathetic. Specifically, we have determined the positive and negative affects of participants using the PANAS scale. Positive and negative affectivity scores can vary from 5 to 25 points. In our study, the mean positive affectivity score, obtained from the sum of positive affects, was high $(M=16.2 ; S D=3.139)$. The mean negative affectivity score of participants was low $(M=6.8 ; S D=2.152)$ (Table 2$)$. According to Watson et al. (1988), a high positive affectivity score is representative of a state of concentration, energy and positive engagement, the low score of negative affectivity reflects a state of serenity and calm. 
Table 2. Summary of the mean results for participants' moods (5-point Likert scale).)

\begin{tabular}{lccc}
\hline & Positive (P) & & \\
Affect & Negative (N) & $\bar{x}$ & $\sigma$ \\
\hline Upset & $\mathrm{N}$ & 1.19 & 0.525 \\
Hostile & $\mathrm{N}$ & 1.17 & 0.550 \\
Alert & $\mathrm{P}$ & 2.67 & 0.964 \\
Ashamed & $\mathrm{N}$ & 1.23 & 0.509 \\
Inspired & $\mathrm{P}$ & 2.67 & 0.923 \\
Nervous & $\mathrm{N}$ & 2.04 & 1.028 \\
Determined & $\mathrm{P}$ & 3.35 & 0.883 \\
Attentive & $\mathrm{P}$ & 3.87 & 0.742 \\
Afraid & $\mathrm{N}$ & 1.17 & 0.474 \\
Active & $\mathrm{P}$ & 3.65 & 0.947 \\
\hline
\end{tabular}

Regarding the participant's empathy score, the results of QCAE questionnaire are high, regardless of expressiveness modalities and situations (affective empathy: $M=$ 33.21 out of max. 48 points; $S D=4.25$; cognitive empathy: $M=57.78$ out of max. 76 points; $S D=3.75)$.

Following the analysis of these results, we have conducted a statistical analysis between the participants' mood and empathy scores obtained from the QCAE questionnaire, to analyze whether there is a possible correlation. We have also taken into account the age of the participants to analyze a potential correlation between age and empathy as mentioned in our state of the art. We first performed the KolmogorovSmirnov test and the Levene test in order to verify the normality and homogeneity of the data distribution in situation 1 and situation 2 . The results of these allowed us to choose the Pearson test for situation 1 (Table 3) and situation 2 (Table 4).

Table 3. Correlation table concerning positive and negative affectivity, age and dimensions of empathy for situation 1 (Positive Affectivity - PA, Negative Affectivity - NA, Perspective Taking - PT, Online Simulation - OS, Emotional Contagion - EC, Proximal Reactivity - PRR, Peripheral Reactivity - PER)

\begin{tabular}{|c|c|c|c|c|c|c|c|c|}
\hline & PA & NA & Age & PT & OS & EC & PRR & PER \\
\hline PA & - & -0.011 & 0.097 & -0.006 & 0.155 & -0.067 & 0.210 & 0.094 \\
\hline NA & & - & -0.023 & -0.137 & -0.083 & 0.248 & 0.068 & -0.145 \\
\hline Age & & & - & -0.186 & $-0.374 * *$ & $-0.444 * *$ & $-0.279 *$ & -0.001 \\
\hline PT & & & & - & $0.345^{*}$ & 0.093 & 0.060 & 0.010 \\
\hline os & & & & & - & $0.422 * *$ & $0.326^{*}$ & 0.217 \\
\hline EC & & & & & & - & $0.519 * *$ & 0.153 \\
\hline PRR & & & & & & & - & $0.422 * *$ \\
\hline PER & & & & & & & & - \\
\hline
\end{tabular}

Our results showed no correlation between empathy and positive or negative affectivity in situation 1 (Table 3). However, we observed negative correlations between age and online simulation rho $=-0.374, n=52, p=0.006$, age and emotional contagion rho $=-0.444, n=52, p=0.001$ and age and proximal reactivity $r h o=-0.279, n=$ $52, p=0.045$. 
Table 4. Correlation table concerning positive and negative affectivity, age and dimensions of empathy for situation 2 (Positive Affectivity - PA, Negative Affectivity - NA, Perspective Taking - PT, Online Simulation - OS, Emotional Contagion - EC, Proximal Reactivity - PRR, Peripheral Reactivity - PER)

\begin{tabular}{|c|c|c|c|c|c|c|c|c|}
\hline & PA & NA & Age & $\mathrm{PT}$ & OS & EC & PRR & PER \\
\hline PA & - & -0.011 & 0.097 & 0.080 & 0.099 & -0.160 & 0.210 & 0.094 \\
\hline NA & & - & -0.023 & -0.233 & 0.054 & 0.108 & 0.068 & -0.145 \\
\hline Age & & & - & -0.167 & $-0.339 *$ & $-0.464 * *$ & $-0.279 *$ & -0.001 \\
\hline PT & & & & - & $0.377 * *$ & 0.175 & 0.221 & 0.213 \\
\hline OS & & & & & - & $0.494 * *$ & $0.464 * *$ & $0.282 *$ \\
\hline EC & & & & & & - & $0.419 * *$ & 0.001 \\
\hline PRR & & & & & & & - & $0.422 * *$ \\
\hline PER & & & & & & & & \\
\hline
\end{tabular}

For the second situation, we also observed negative correlations between age and online simulation rho $=-0.339, n=52, p=0.014$, age and emotional contagion rho $=-0.464, n=52, p=0.001$ and age and proximal reactivity $r h o=-0.279, n=52, p$ $=0.045$ (Table 4).

In both situations, there are correlations between the participant's age and empathy score: the older the participant is, the lower the empathy score is.

\subsection{Impact of the facial expressiveness of virtual agents on user empathy}

In order to determine whether the expressiveness of the agents impacts the empathy of the users, we performed an inferential analysis. We used the Kolmogorov-Smirnov test and the Levene test to verify the normality and homogeneity of the data distribution. Levene's test did not reject the hypothesis of variance homogeneity. Therefore, we used parametric tests. We performed t-tests for independent samples. Results are considered significant when $p<0.05$. We did not consider items related to empathy in the user's daily life because they are not contextualized. They are therefore not adapted to the case of use. These items correspond to 3 items from the perspectivetaking and the items of the proximal and peripheral reactivity dimensions. We have therefore considered only 7 of the 10 items from the perspective analysis (PT-7) in this analysis.

We analyzed the impact of the virtual agent's facial expressiveness on empathy in situation 1. The comparison of empathy scores was made between the group of participants $(\mathrm{N}=26)$ using the simulator with a realistic and expressive virtual agent (EVA) and the group $(\mathrm{N}=26)$ using the simulator with the same realistic virtual agent, but without any facial expressiveness (VA).

Concerning the first situation, our results revealed a significant difference concerning perspective-taking (PT-7), a component of cognitive empathy, in favour of the expressive virtual agent (EVA) $(Z=2.280 ; p=0.027)$. It should be noted that the expressiveness of the agent impacts the cognitive empathy of the user (Table 5). 
Table 5. Impact of the facial expressiveness of virtual agents on the dimensions of empathy

\begin{tabular}{|c|c|c|c|c|c|}
\hline & \multicolumn{2}{|c|}{ EVA } & \multicolumn{2}{|c|}{ VA } & \multirow[t]{2}{*}{$\mathrm{p}$} \\
\hline & $\bar{x}$ & $\sigma$ & $\bar{x}$ & $\sigma$ & \\
\hline Perspective taking (PT-7) & 19,08 & 1,671 & 18,04 & 1,612 & 0.027 \\
\hline Online Simulation (OS) & 30,81 & 2,684 & 30,38 & 2,858 & \\
\hline Emotional contagion (EC) & 9,77 & 2,471 & 8,81 & 2,546 & \\
\hline
\end{tabular}

We used the same statistical tests for the second situation. However, we did not observe significant differences in the impact of expressiveness on user empathy in this situation. These initial results suggest that the contexts of the two situations impact the user's empathy. Therefore, we compared the empathy of the participants in situation 1 and situation 2. We used the Kolmogorov-Smirnov test to check for normality of distribution and the Levene test for data homogeneity. The data followed a normal distribution ( $p>0.05$ for the majority of the tested variables) and Levene's test did not reject the hypothesis of variance homogeneity. Therefore, we performed t-tests for independent samples. The results are considered significant when $p<0.05$.

There were significant differences in the simulation in favour of situation 1 for participants interacting with the EVA $(Z=2.917 ; p=0.005)$ and for participants interacting with the VA $(Z=2.323 ; p=0.024)$, and a significant difference for the VA in favour of situation $2(Z=3,133 ; p=0.003)$. These results demonstrate the impact of context on user empathy and we assume it is related to the nature of the interactions. In situation 1, the participants were encouraged to reflect on the most appropriate questions to ask the patient. They have limited information about her emotional state and have to imagine themselves in her place. In contrast to situation 1, situation 2 consisted principally of predefined dialogues in which the patient expresses her discomfort. The learners were mainly in a spectator position during situation 2, they did not need to be attentive to the patient's emotional reactions or to imagine her emotional state, because it was indicated directly to them without any particular action on their part. Moreover, with the predefined exchanges, they had no choice as to how they respond to the patient.

In summary, the expressiveness of the agent have promoted cognitive empathy, especially the dimension of perspective taking in situation 1. However, the impact of the context must be taken into consideration, as it may influence the empathic reactions of the users (De Vignemont \& Singer, 2006; Paiva et al., 2017).

\subsubsection{Impact of facial expressiveness of virtual agents on imitation and emotional contagion}

During the experimentation, we captured the facial expressions of the 52 participants. However, we removed a participant for whom facial expression capture was not activated due to a webcam malfunction. Among the remaining 51 participants, 27 participants had emotional reactions within situation 1 and 26 participants had emotional reactions within situation 2. This means that the Affdex plugin indicates that it captured a facial expression during the exchanges with the virtual patient. To limit errors, we have set a threshold of intensity at $10 \%$, we did not take into account facial expressions below this threshold. There was a total of 70 emotional reactions during the exchanges in situation 1, we wanted to consider these responses to understand whether they tended to be imitative. For the participants who were confronted with 
the EVA in situation 1, there were 10 potential facial imitations out of 27 reactions, i.e. the participant reacted with the same emotion as that expressed by the patient, based on the capture of facial expressions. For example, during the first exchange with the realistic and expressive patient, the patient greeted the user, smiled and indicated that she was happy to see him. During this exchange, we listed 6 participants who had an emotional facial expression, including 4 who had an expression of joy, with an intensity varying from 14 to $81 \%$ depending on the participants. Despite the potential interest of these data, they are not sufficient, on their own, to perform a reliable analysis. It would be interesting to perform new facial expression captures coupled with psycho-physiological measures.

\subsection{Sentiment of similarity and affective bond}

We analyzed the impact of the agents' facial expressiveness on the user's sense of similarity and affective bond with the agent. These dimensions were obtained by analyzing the responses to the two items regarding the feeling of similarity, and the response to the item concerning a potential affectivity towards the agent (e.g. 3.6 Measure). The Kolmogorov-Smirnov test was used to verify the normality of the data distribution for situation 1 as well as for situation 2. The data did not follow a normal distribution $(p<0.05)$. In order to compare the scores for the feeling of similarity and the development of an affective bond according to the expressiveness of the agent, we used the Mann-Whitney test. The tests did not reveal any significant differences on the items regarding the feeling of similarity and the development of an affective bond between EVA and VA in situation 1. The tests also revealed no significant differences on these items between EVA and VA in situation 2. The patient's facial expressiveness did not seem to affect affectivity and similarity.

We assume that the participants considered the patient in her entirety. The impact of facial expressiveness was slight compared to the overall emotional context provided by the patient and the simulator. However, the mean scores related to the feeling of similarity and affective bond were high ( $M>4$ on a 5-point Likert scale for all 3 items), regardless of the expressiveness of the agent. It suggests that each modality potentially allows the development of an affective bond and a feeling of similarity. This confirms that the characteristics and the relationship established with the virtual agent are important elements for the user.

On average, the participants reported that they were comfortable with the virtual patient and that her reactions seemed realistic (situation 1: $M=4.29 ; S D=0.696$ ; situation $2: M=4.08 ; S D=0.763)$. Participants also indicated that the patient looks or might look like one of their patients (situation 1: $M=4.13 ; S D=0.864$; situation $2: M=4.12 ; S D=0.90$ ). In addition, on average, the participants were able to develop affection for the patient, and most of them enjoyed the interaction with her (situation 1: $M=4.33 ; S D=0.648$; situation $2: M=4.21 ; S D=0.723$ ).

\subsection{Post-experiment questionnaire}

We conducted a statistical analysis to compare the participants' feelings about: their emotional involvement with the virtual patient, the virtual patient's expressiveness, and the empathy felt for the virtual patient in situation 1 and situation 2 . The table below contains the means of the participants' responses, evaluated with 5-point Likert scales $(1=$ Strongly disagree, $5=$ Strongly agree). 
In order to conduct an inferential analysis and to compare these data, the Kolmogorov-Smirnov test was carried out to verify the normality of the distribution of responses to the post-processing questionnaire. The data did not follow a normal distribution $(p<0.05$ for all tested variables). We used non-parametric tests, the Mann Whitney test. However, there were no significant differences regarding the expressiveness of virtual agents.

Table 6. Summary of participants' responses to the post-experiment questionnaire

\begin{tabular}{lcccc}
\hline & \multicolumn{2}{c}{ EVA } & \multicolumn{2}{c}{ VA } \\
Situation 1 & $\bar{x}$ & $\sigma$ & $\bar{x}$ & $\sigma$ \\
\hline Emotional involvement & 3.58 & 1.027 & 3.58 & 0.857 \\
Patient expressiveness & 3.81 & 1.059 & 3.27 & 1.282 \\
Empathy & 3.92 & 1.017 & 3.85 & 0.925 \\
\hline Situation 2 & & & & \\
\hline Emotional involvement & 3.73 & 0.827 & 3.62 & 1.023 \\
Patient expressiveness & 3.62 & 0.941 & 3.38 & 1.203 \\
Empathy & 4.00 & 0.693 & 3.96 & 0.871 \\
\hline
\end{tabular}

Finally, participants indicated that they felt this type of simulator would be beneficial for nursing education $(M=4.42 ; S D=0.696)$. In addition, they appreciated interacting with the virtual agent. It is also interesting to note that some participants referred to the virtual patient as if she was a real patient, attributing personality, behaviours and feelings to her:

"She was a little more embarrassed in situation 1 because she didn't know me, I guess."

"Yes, she was nicer at first. And a little worried at the end."

This confirms that participants had a major interest in this health simulation and its virtual patient. Moreover, they noted that the expressiveness of the virtual patient was an important element in the design of a nursing simulator $(M=4.5$; $S D=0.728)$. However, several participants stated that they would have liked virtual patients to be more expressive, they indicated:

" [...] The patient's expression is not sufficient for my taste."

"It can be rewarding for people starting in the profession to conceptualize toileting and first contact with a patient and the professional postures to adopt, but perhaps you need to visualize the patient more with her emotions.

"(You should) add more facial expressions, facial expressions, gestures"

"I think it would be interesting to improve facial and body expressions even further, I missed it to experience emotions..." 


\section{Discussion}

\subsection{Empathy}

In line with previous research on empathy and virtual agents (De Rosis, Cavalluzzi, Mazzotta, \& Novielli, 2005; Deladisma et al., 2007; Paiva et al., 2005; Volonte et al., 2016), the results of our experiment concerning the participant's empathy score is high, regardless of expressiveness modalities and situations. This high level of empathy assumes that the participants are emotionally involved in the simulation. This involvement is indicative of a strong emotional context emanating from the simulator and the virtual agent. According to the literature, emotional involvement promotes memorization and leads to better learning (Tyng et al., 2017; Veletsianos, 2009).

Based on our literature review, user characteristics, such as mood and age, have an impact on empathy (De Vignemont \& Singer, 2006; Lennon \& Eisenberg, 1987; Paiva et al., 2017). According to a study of medical interns, a calm mood promotes empathy towards a patient, whereas stress, for example, does not promote the induction of empathy (Joubert, 2014). In this sense, our results show a high positive affectivity score, signifying concentration and energy, and a low negative affectivity score, representative of a state of serenity and calm (Watson et al., 1988). We don't observe a correlation between the empathy score and positive and negative affectivity regardless of the situation. However, we observe an impact of age on the empathy score. Our results indicate a negative correlation between participant age and the dimensions of emotional contagion, online simulation and proximal reactivity. The older the participant is, the lower the empathy score is. The oldest students of the Nursing Institutes are generally in professional reorientation. This mainly concerns care assistants and childcare assistants. Regarding age, we assume that the professional background and experience of this kind of participants may have influenced their emotional reactions to the virtual patient.

\subsubsection{Impact of facial expressiveness of virtual agents on the induction of empathy}

There is a relation between the expressiveness of virtual agents and user empathy, as has been highlighted in research on human interactions (Dimberg, Thunberg, \& Elmehed, 2000; Drimalla, Landwehr, Hess, \& Dziobek, 2019; Jospe, Flöel, \& Lavidor, 2018). In line with the implementation of an empathic situation described by Paiva et al. (2017), our results reveal a significant difference on the user's perspective-taking, a component of cognitive empathy, in favor of the virtual agent endowed facial expressiveness.

In situation 1, the perspective-taking is more significant when the user is in front of the expressive virtual agent than with a virtual agent without facial expressiveness. This difference indicates that the facial expressiveness of the virtual agent impacts the user, who tends to adopt spontaneously the psychological point of view of the virtual patient. These results corroborate our hypothesis 1.1. Paiva et al. (2017) indicate, in their research, that the degree of expressiveness of the virtual agent is important because it is a fundamental indicator of the empathy process; and that this aspect is essential if the virtual agent represents a virtual human. Indeed, the results of our study show that participants are slightly more empathetic when they are confronted with virtual agents with emotional facial expressions.

However, it is recommended to take in consideration the difference in the context of the study, as these are factors that can influence empathic reactions (De Vignemont 
\& Singer, 2006). This is corroborated by the comparative study of the induction of empathy between our two situations, to the detriment of the expressiveness of the virtual agent. It would be interesting to conduct further studies on the impact of context on the induction of empathy in simulator users, especially about the different types of interactions (predefined dialogues or choice of questions), the scenario, the narration or the duration of the simulation.

\subsubsection{Impact of facial expressiveness of virtual agents on imitation and emotional contagion}

Concerning the impact of expressiveness on the emotional contagion evaluated using the QCAE questionnaire, we do not observe significant differences between the expressive virtual agent (EVA) and the virtual agent (VA). In addition, during our analysis of the emotional reactions resulting from the capture of facial expressions, it appears that some participants had emotional reactions consistent with the emotional states expressed by the virtual agent, particularly the expression of joy. These results are in line with previous research on users' emotional reactions to emotional stimuli (Schrammel, Pannasch, Graupner, Mojzisch, \& Velichkovsky, 2009; Weyers et al., 2006). Advanced analyses could indicate whether this is imitation or emotional contagion. However, as discussed above, due to the low precision of these data and the absence of significant differences in the QCAE questionnaire regarding emotional contagion, we are not currently able to confirm an impact of the facial expressiveness of virtual agents on affective empathy, we are not able to confirm our hypothesis 1.2.

In their work on the impact of emotional facial expressions of virtual agents, Ravaja et al. (2016) highlighted the influence of expressiveness on the user's emotional responses (facial muscle activity), particularly through psychophysiological measures: electromyography (EMG) and electroencephalography (EEG). This is also the case in the recent study by Philip et al. (2018), where virtual agents are used to generate controlled emotional stimuli to induce rapid facial responses in the user, including the emotion of anger and joy. It would be interesting to conduct an additional study with advanced measures of affective empathy such as physiological measures recommended in various previous research studies (Dimberg, Andréasson, \& Thunberg, 2011; Neumann, Chan, Boyle, Wang, \& Westbury, 2015; Neumann \& Westbury, 2011).

In regards to our results, it would also be relevant to conduct a complementary study on the representation of emotions. In a strong emotional context (scenario, context, dialogue, etc.) and a panel not very familiar with simulators, it is complex to determine whether facial expressiveness must be identical to that of a real human or whether it can be intensified to be perceived as effectively as on a human. Intensification could provoke greater empathic reactions in the user, while avoiding the Uncanny Valley phenomenon as discussed in the research work of Mäkäräinen, Kätsyri, and Takala (2014). The facial expressiveness of the virtual agent in our study does not impact affective empathy, suggesting that the representation of emotions is probably too weak to cause a real emotional contagion and imitation.

\subsection{Sentiment of similarity and affective bond}

Our results do not reveal any significant difference in the feeling of similarity or potentially developed affective bond with the patient, depending on the facial expressiveness of the virtual agents. We are therefore not able to confirm the H2 hypothesis. However, our results corroborate the previous observations of Hall et al. (2005); Vugt, Bailen- 
son, Hoorn, and Konijn (2010), the mean results observed for each of the questions on affective bond and similarity present values close to the maximum on our scale (greater than 4 on 5). These findings seem to demonstrate the possibility for a user to experience a sense of similarity and affection towards the virtual agent (regardless of facial expression). This confirms that there is a major interest for the virtual agent in this simulation.

Many participants have appreciated interacting with the virtual patient. The virtual agent and their exchanges are important for the user. It is interesting to note that some participants referred to the virtual patient as a real person. This confirms that there is a major interest in the virtual agent in this simulation with its strong emotional context. It seems that the participants were able to get past the exercise, bypassing the technology and the agent to focus on the patient, her needs, and her care.

\subsection{Feedback on nursing simulators}

Responses to the post-experiment questionnaire have confirmed that on average, participants believe that the simulators in nursing education is beneficial. Also, they have reported that the expressiveness of the virtual patient is an important element in the use of simulators.

\subsection{Critical Analysis}

As this study only concerns the care of an elderly patient, it would be relevant to repeat the study with male agents of various ages in order to take into account possible differences in sex and age. The main limit of our experimentation concerns the strong emotional context of this simulation, due to the scenario, the environments, the visual and behavioural realism of the virtual agents, the dialogues and interactions. We assume that the expressiveness of the virtual agent is too weak to be noticeable with the set of factors soliciting the users' attention within the simulator. The concentration of participants is mainly on their medical knowledge and little on the degree of expressiveness of the virtual agent. In this strong emotional context and the demand for nursing knowledge, the impact of facial expressiveness on empathy can be limit. As mention above, intensifying facial expressions would be beneficial in measuring the impact of expressiveness on empathy.

\section{Conclusion}

Our results show a high score on the empathy questionnaire among participants who take charge virtual patients. Depending of the context, the facial expressiveness of realistic virtual agents impacts the cognitive empathy of the user. It slightly encourages users to adopt the point of view of the patient, i.e. perspective-taking.

This study identifies the potentialities induced by virtual agent's emotional facial expressions on the empathy induction in the user of nursing simulators. This contributes to recommendations regarding the factors of empathy in virtual environments. It also aims to improve health simulation experiences. Indeed, the induction of emotional responses in the learner and greater emotional involvement are effective ways to improve content memorization and lead to better learning.

Following the analysis of users' responses and feelings, we assume that it would be 
possible to improve this result for cognitive and affective empathy, in particular by intensifying the facial emotional expressions expressed by virtual agents.

\section{Acknowledgments}

We would like to thank our laboratory and its team for their advice and valuable suggestions throughout our work. We would also like to thank the participants and the Nursing Institutes for their interest and availability. We extend our thanks to SimforHealth for funding this research.

\section{References}

Baron-Cohen, S., \& Wheelwright, S. (2004). The empathy quotient: an investigation of adults with Asperger syndrome or high functioning autism, and normal sex differences. Journal of autism and developmental disorders, 34(2), 163-175.

Bates, J. (1994). The role of emotion in believable agents. Communications of the ACM, Special Issue on Agents.

Baylor, A. L., \& Kim, S. (2009). Designing nonverbal communication for pedagogical agents: When less is more. Computers in Human Behavior, 25(2), 450-457.

Beale, R., \& Creed, C. (2009). Affective interaction: How emotional agents affect users. International Journal of Human-Computer Studies, 67(9), 755-776.

Berthoz, A., \& Jorland, G. (2004). Empathie (L'). Odile Jacob.

Boukricha, H. (2013). Simulating empathy in virtual humans (Unpublished doctoral dissertation). Bielefeld University.

Chaturvedi, H., Newsome, N. D., \& Babu, S. V. (2015). An evaluation of virtual human appearance fidelity on user's positive and negative affect in human-virtual human interaction. 2015 IEEE Virtual Reality (VR), 163-164.

Courgeon, M., Buisine, S., \& Martin, J.-C. (2009). Impact of expressive wrinkles on perception of a virtual character's facial expressions of emotions. In 9th international conference iva, netherlands (pp. 201-214).

Davis, M. H. (1983). Measuring individual differences in empathy: Evidence for a multidimensional approach. Journal of personality and social psychology, 44(1), 113.

De Melo, C. M., \& Gratch, J. (2009). Expression of emotions using wrinkles, blushing, sweating and tears. In Proceedings of the international conference iva (pp. 188-200).

De Rosis, F., Cavalluzzi, A., Mazzotta, I., \& Novielli, N. (2005). Can embodied conversational agents induce empathy in users? Virtual Social Agents, 65.

De Vignemont, F., \& Singer, T. (2006). The empathic brain: how, when and why? Trends in cognitive sciences, 10(10), 435-441.

Decety, J. (2002). Naturaliser l'empathie. L'encéphale, 28(1), 1-12.

Deladisma, A. M., Cohen, M., Stevens, A., Wagner, P., Lok, B., Bernard, T., ... Lind, D. S. (2007). Do medical students respond empathetically to a virtual patient? American Journal of Surgery, 193(6), 756-760.

Dimberg, U., Andréasson, P., \& Thunberg, M. (2011). Emotional empathy and facial reactions to facial expressions. Journal of Psychophysiology.

Dimberg, U., Thunberg, M., \& Elmehed, K. (2000). Unconscious Facial Reactions to Emotional Facial Expressions. Psychological Science, 11(1), 86-89.

Drimalla, H., Landwehr, N., Hess, U., \& Dziobek, I. (2019). From face to face: the contribution of facial mimicry to cognitive and emotional empathy. Cognition and Emotion, 1-15.

Ekman, P. (1993). Facial expression and emotion. American psychologist, 48(4), 384.

Ekman, P. (2007). Emotions revealed: Recognizing faces and feelings to improve communication and emotional life (Vol. 12). Macmillan. 
Ekman, P., \& Friesen, W. V. (1978). Manual for the facial action coding system. Consulting Psychologists Press.

Fabri, M., \& Moore, D. (2005). The use of emotionally expressive avatars in collaborative virtual environments. Virtual Social Agents, 88(April), 88-94.

Frith, C., \& Frith, U. (1999). Interacting minds-a biological basis. Science, 286(5445), $1692-1695$.

Frith, C., \& Frith, U. (2005). Theory of mind. Current Biology, 15(17), R644-R645.

Garau, M. (2003). The impact of avatar fidelity on social interaction in virtual environments (Unpublished doctoral dissertation). University of London.

Glas, N., \& Pelachaud, C. (2015). Definitions of engagement in human-agent interaction. In Proceedings of the workshop on engagement in social intelligent virtual agents. (p. 33-40).

Guadagno, R., Blascovich, J., Bailenson, J., \& McCall, C. (2007). Virtual humans and persuasion: The effects of agency and behavioral realism. Media Psychology, 10(1), 1-22.

Guillain, A., \& Pry, R. (2012). D'un miroir l'autre. Fonction posturale et neurones miroirs. Bulletin de psychologie, 518(2), 115-127.

Guo, F., Mingming, L., Qingwing, Q., \& Duffy, V. (2019). The effect of a humanoid robot's emotional behaviors on users' emotional responses: Evidence from pupillometry and electroencephalography measures. International Journal of Human-Computer Interaction, 35.

Hall, L., Woods, S., Aylett, R., Newall, L., \& Paiva, A. (2005). Empathic interaction with synthetic characters: the importance of similarity. Encyclopaedia of Human Computer Interaction.

Hasler, B. S. B. S., Spanlang, B., \& Slater, M. (2017). Virtual race transformation reverses racial ingroup bias. PLoS ONE, 12(4), 1-20.

Hess, U., \& Fischer, A. (2014). Emotional mimicry: Why and when we mimic emotions. Social and Personality Psychology Compass, 8(2), 45-57.

Hoffman, M. L. (2001). Empathy and moral development: Implications for caring and justice. Cambridge University Press.

Iacoboni, M. (2009). Imitation, Empathy, and Mirror Neurons. Annual Review of Psychology, $60(1), 653-670$.

Jospe, K., Flöel, A., \& Lavidor, M. (2018). The interaction between embodiment and empathy in facial expression recognition. Social cognitive and affective neuroscience, 13(2), 203-215.

Joubert, A. (2014). Étude qualitative des déterminants de l'empathie chez les internes en médecine générale (Unpublished doctoral dissertation). Université Paris Descartes.

Larboulette, C., \& Cani, M.-P. (2004). Real-time dynamic wrinkles. In Computer graphics international, 2004. proceedings (pp. 522-525).

Lemoine, J.-F., \& Cherif, E. (2012). Comment générer de la confiance envers un agent virtuel à l'aide de ses caractéristiques? Une étude exploratoire. Management $\&$ Avenir, 1(8).

Lennon, R., \& Eisenberg, N. (1987). Gender and age differences in empathy and sympathy. Empathy and its development, 195-217.

Li, Z., Mao, X., \& Liu, L. (2009). Providing expressive eye movement to virtual agents. Proceedings of the international conference on Multimodal interfaces - ICMI, 241.

Liang, Y.-s., Yang, H.-x., Ma, Y.-t., Lui, S. S. Y., Cheung, E. F. C., Wang, Y., \& Chan, R. C. K. (2019). Validation and extension of the questionnaire of cognitive and affective empathy in the Chinese setting. PsyCh journal, 8(4), 439-448.

MacDonald, A. (2003). I feel your pain (and joy): New theories about empathy. Brain Work, 13(4), 1-3.

Mäkäräinen, M., Kätsyri, J., \& Takala, T. (2014). Exaggerating facial expressions: A way to intensify emotion or a way to the uncanny valley? Cognitive Computation, 6(4), 708-721.

McDonnell, R., Breidt, M., \& Bülthoff, H. H. (2012). Render me Real? Investigating the Effect of Render Style on the Perception of Animated Virtual Humans. ACM Transactions on Graphics, 31(4), 1-11.

McDuff, D., Mahmoud, A., Mavadati, M., Amr, M., Turcot, J., \& el Kaliouby, R. (2016). AFFDEX SDK: a cross-platform real-time multi-face expression recognition toolkit. In Proceedings of the 2016 chi conference extended abstracts on human factors in computing 
systems (pp. 3723-3726).

Milcent, A.-S., Geslin, E., Kadri, A., \& Richir, S. (2019). Expressive Virtual Human: Impact of expressive wrinkles and pupillary size on emotion recognition. In Proceedings of the 19th acm international conference on intelligent virtual agents (pp. 215-217).

Mori, M., MacDorman, K. F., \& Kageki, N. (2012). The uncanny valley [from the field]. IEEE Robotics \& Automation Magazine, 19(2), 98-100.

Mori, M., MacDorman, K. F. t., \& Minato, T. t. (1970). The Uncanny Valley. Energy, 7(4), 33-35.

Myszkowski, N., Brunet-Gouet, E., Roux, P., Robieux, L., Malézieux, A., Boujut, E., \& Zenasni, F. (2017). Is the Questionnaire of Cognitive and Affective Empathy measuring two or five dimensions? Evidence in a French sample. Psychiatry research, 255, 292-296.

Narme, P., Mouras, H., Loas, G., Krystkowiak, P., Roussel, M., Boucart, M., \& Godefroy, O. (2010). Vers une approche neuropsychologique de l'empathie. Revue de neuropsychologie, 2(4), 292-298.

Neumann, D. L., Chan, R. C. K., Boyle, G. J., Wang, Y., \& Westbury, H. R. (2015). Measures of empathy: Self-report, behavioral, and neuroscientific approaches. In Measures of personality and social psychological constructs (pp. 257-289). Elsevier.

Neumann, D. L., \& Westbury, H. R. (2011). The psychophysiological measurement of empathy. Psychology of empathy, 119-142.

Ochs, M., Niewiadomski, R., \& Pelachaud, C. (2015). Facial Expressions of Emotions for Virtual Characters. The Oxford Handbook of Affective Computing, 261.

Paiva, A., Dias, J., Sobral, D., Aylett, R., Woods, S., Hall, L., \& Zoll, C. (2005). Learning by feeling: Evoking empathy with synthetic characters. Applied Artificial Intelligence, 19(3-4), $235-266$.

Paiva, A., Dias, J., Sobral, D., Woods, S., \& Hall, L. (2004). Building empathic lifelike characters: the proximity factor. In Workshop on empathic agents, aamas (Vol. 4).

Paiva, A., Leite, I., Boukricha, H., \& Wachsmuth, I. (2017). Empathy in Virtual Agents and Robots: A Survey. ACM Transactions on Interactive Intelligent Systems (TiiS), 7(3), 11.

Pelachaud, C. (2009). Modelling multimodal expression of emotion in a virtual agent. Philosophical Transactions of the Royal Society B: Biological Sciences, 364(1535), 3539-3548.

Perron, B. (2005). Jeu vidéo et émotions. Le game design de jeux vidéo. Approches de l'expression vidéo-ludique, Éditions Mnémos.

Philip, L., Martin, J.-C., \& Clavel, C. (2018). Rapid Facial Reactions in Response to Facial Expressions of Emotion Displayed by Real Versus Virtual Faces. i-Perception, 9(4), 1-18.

Queirós, A., Fernandes, E., Reniers, R., Sampaio, A., Coutinho, J., \& Seara-Cardoso, A. (2018). Psychometric properties of the questionnaire of cognitive and affective empathy in a Portuguese sample. PloS one, 13(6).

Ravaja, N., Bente, G., Kätsyri, J., Salminen, M., \& Takala, T. (2016). Virtual character facial expressions influence human brain and facial EMG activity in a decision-making game. IEEE Transactions on Affective Computing, 9(2), 285-298.

Reniers, R. L. E. P., Corcoran, R., Drake, R., Shryane, N. M., \& Völlm, B. A. (2011). The QCAE: A questionnaire of cognitive and affective empathy. Journal of personality assessment, 93(1), 84-95.

Rizzolatti, G., \& Sinigaglia, C. (2008). Neurones miroirs (Vol. 29). Odile Jacob.

Rodrigues, S. H., Mascarenhas, S., Dias, J., \& Paiva, A. (2015). A process model of empathy for virtual agents. Interacting with Computers, 27(4), 371-391.

Rodrigues, S. H., Mascarenhas, S. F., Dias, J., \& Paiva, A. (2009). "I can feel it too!" : Emergent empathic reactions between synthetic characters. In 20093 rd international conference on affective computing and intelligent interaction and workshops (pp. 1-7).

Schrammel, F., Pannasch, S., Graupner, S.-T., Mojzisch, A., \& Velichkovsky, B. M. (2009). Virtual friend or threat? The effects of facial expression and gaze interaction on psychophysiological responses and emotional experience. Psychophysiology, 46(5), 922-931.

Ter Stal, S., Tabak, M., Op Den Akker, H., Tessa, B., \& Hermie, H. (2020). Who do you prefer? The effect of age, gender and role on users' first impressions of embodied conversational 
agents in eHealth. International Journal of Human-Computer Interaction, 36, no 9, $p$. 881-892.

Thompson, E. R. (2007). Development and validation of an internationally reliable short-form of the positive and negative affect schedule (PANAS). Journal of cross-cultural psychology, $38(2), 227-242$.

Tyng, C. M., Amin, H. U., Saad, M. N. M., \& Malik, A. S. (2017). The Influences of Emotion on Learning and Memory. Frontiers in Psychology, 8.

Veletsianos, G. (2009). The impact and implications of virtual character expressiveness on learning and agent-learner interactions. Journal of Computer Assisted Learning, 25(4), $345-357$.

Vinayagamoorthy, V., Gillies, M., Steed, A., Tanguy, E., Pan, X., Loscos, C., \& Slater, M. (2006). Building Expression into Virtual Characters. The Eurographics Association, 1-42.

Volonte, M., Babu, S. V., Chaturvedi, H., Newsome, N., Ebrahimi, E., Roy, T., ... Fasolino, T. (2016, apr). Effects of Virtual Human Appearance Fidelity on Emotion Contagion in Affective Inter-Personal Simulations. IEEE Transactions on Visualization and Computer Graphics, 22(4), 1326-1335.

Von Der Pütten, A. M., Krämer, N. C., \& Gratch, J. (2009). Who's there? Can a Virtual Agent Really Elicit Social Presence? The 12th Annual International Workshop on Presence.

Vugt, H. C. V., Bailenson, J. N., Hoorn, J. F., \& Konijn, E. A. (2010). Effects of facial similarity on user responses to embodied agents. ACM Transactions on Computer-Human Interaction (TOCHI), $17(2), 7$.

Watson, D., Clark, L. A., \& Tellegen, A. (1988). Development and validation of brief measures of positive and negative affect: the PANAS scales. Journal of personality and social psychology, 54(6), 1063.

Weyers, P., Mühlberger, A., Hefele, C., \& Pauli, P. (2006). Electromyographic responses to static and dynamic avatar emotional facial expressions. Psychophysiology, 43(5), 450-453.

Wicker, B., Keysers, C., Plailly, J., Royet, J.-P., Gallese, V., \& Rizzolatti, G. (2003). Both of us disgusted in My insula: the common neural basis of seeing and feeling disgust. Neuron, 40(3), 655-664.

Zell, E., Aliaga, C., Jarabo, A., Zibrek, K., Gutierrez, D., McDonnell, R., \& Botsch, M. (2015). To stylize or not to stylize?: the effect of shape and material stylization on the perception of computer-generated faces. ACM Transactions on Graphics (TOG), 34(6), 184.

Zell, E., Zibrek, K., \& McDonnell, R. (2019). Perception of virtual characters. In Acm siggraph 2019 courses (p. 21).

Zibrek, K., Martin, S., \& McDonnell, R. (2019). Is Photorealism Important for Perception of Expressive Virtual Humans in Virtual Reality? ACM Transactions on Applied Perception (TAP), $16(3), 14$.

\section{Biographies}

Anne-Sophie Milcent, Ph.D. Her research work focuses on expressive virtual agents and their impact on the user experience, with an application to the field of medical education.

Abdelmajid Kadri, Ph.D in computer science specializing in virtual technologies. His research work focuses on the contributions of virtual technologies $(\mathrm{VR}, \mathrm{AR}, \ldots)$ in different application domains, particularly in product design to respond to the needs of design assistance, as well as in health (awareness and rehabilitation).

Simon Richir, M.Eng, Ph.D., Professor at ENSAM (Arts et Metiers), he is also the head of "Laval Arts et Metiers Institute" and "Presence innovation" research team (LAMPA Lab). His research and teaching activities concentrate on technological innovation, engineering design process, ideation, innovative projects, and innovative uses of new advanced technologies such as Virtual Reality or Augmented Reality. 